La Revue

des Droits

de l'Homme

\section{La Revue des droits de l'homme}

Revue du Centre de recherches et d'études sur les droits fondamentaux

Actualités Droits-Libertés | 2019

\title{
Le parcours des étrangers malades en France
}

À propos des rapports de l'OFII (13 novembre 2018) et de la CIMADE (16 novembre 2018)

Aude Fraysse, Esther Laneelle, Yasmine Hammadi, Clothilde Lagorsse and Rita Mben

\section{OpenEdition \\ Journals}

Electronic version

URL: http://journals.openedition.org/revdh/5977

DOI: $10.4000 /$ revdh.5977

ISSN: 2264-119X

Publisher

Centre de recherches et d'études sur les droits fondamentaux

\section{Electronic reference}

Aude Fraysse, Esther Laneelle, Yasmine Hammadi, Clothilde Lagorsse and Rita Mben, « Le parcours des étrangers malades en France », La Revue des droits de l'homme [Online], Actualités Droits-Libertés, Online since 18 January 2019, connection on 10 December 2020. URL : http://

journals.openedition.org/revdh/5977 ; DOI : https://doi.org/10.4000/revdh.5977

This text was automatically generated on 10 December 2020 .

Tous droits réservés 


\title{
Le parcours des étrangers malades en France
}

\author{
À propos des rapports de l'OFII (13 novembre 2018) et de la CIMADE (16
} novembre 2018)

\author{
Aude Fraysse, Esther Laneelle, Yasmine Hammadi, Clothilde Lagorsse \\ and Rita Mben
}

1 La loi votée le 7 mars 2016 applicable au 1er janvier 2017 a modifié de manière considérable la situation des étrangers malades. Elle confie au service médical de l'office français de l'immigration et de l'intégration (OFII), composé d'un collège de trois médecins, le soin de donner un avis sur le certificat médical présenté par le demandeur de titre de séjour. Ce collège se prononce sur la nécessité des soins en France et transmet son avis au préfet qui prend la décision finale à la lumière de cet avis. À noter que les médecins faisant désormais partie de l'OFII, lui-même directement lié au ministre de l'Intérieur, le rôle consultatif des Agences régionales de Santé (ARS) s'en trouve affaibli. Ensuite, la loi du 7 mars 2016 a également changé la condition relative à l'accessibilité des soins dans le pays d'origine. À présent, les médecins de l'OFII fondent leur appréciation sur l'offre de soins ainsi que sur les caractéristiques du système de santé dans le pays dont l'étranger est originaire, et non plus sur la seule disponibilité des traitements dans ce pays. À la suite des différentes modifications opérées et de leur mise en œuvre complexe, deux rapports ont examiné l'impact de cette loi sur les étrangers en demande de titres de séjour afin d'être soignés en France.

2 En juin 2018, le Comité inter-mouvements auprès des évacués (CIMADE) a ainsi rendu son bilan après une année passée à observer les effets de la loi sur le statut des étrangers demandeurs de titre de séjour pour soins. Le 13 novembre 2018, l'OFII a, à son tour, publié discrètement ${ }^{1}$ son propre bilan. Ces deux rapports sont sans appel. La loi priorise la lutte contre la fraude au détriment de la prise en charge des situations précaires des étrangers malades. À défaut de disposer d'une carte pluriannuelle, le demandeur doit affronter une procédure de demande digne d'un parcours du combattant, la négation de certaines pathologies ou encore le durcissement de la condition de fond relative à l'accessibilité des soins dans le pays d'origine. En bout de course, les conséquences sont lourdes tant pour 
les personnes étrangères gravement malades que pour les enjeux de santé publique. Les deux rapports, lus en parallèle, mettent en évidence que la loi de 2016 a engendré une diminution des avis favorables (I), notamment liée à la complexification de la procédure (II). L'obsession de lutte contre la fraude (III) a de surcroît conduit l'OFII à ignorer le problème des malades en centre de rétention (IV), une des raisons pour laquelle cette loi avait pourtant été votée.

\section{I/ - L'évolution du droit au séjour pour soins}

3 Avec l'épidémie du SIDA dans les années 80, de nombreux étrangers gravement malades sur le territoire français ont fait l'objet de mesures de renvoi dans leur pays d'origine. Au début des années 1990, plusieurs associations ont alors interpelé le ministère de l'Intérieur sur le cas d'étrangers atteints du VIH soumis à des expulsions. Le ministre de l'Intérieur s'est alors tourné vers le ministre de la Santé. En 1993, Bernard Kouchner, alors ministre de la Santé et de l'action humanitaire, avait exigé la garantie que les personnes concernées par les expulsions pourraient continuer leur traitement dans leur pays d'origine. Afin d'éviter une condamnation pénale pour mise en danger de la vie d'autrui, le ministère de l'Intérieur a alors suivi l'avis du ministère de la Santé. Les préfets, le ministère de l'Intérieur et les médecins se trouvent depuis contraints de travailler ensemble sur la question des étrangers malades. Initialement, un médecin de l'ARS (service déconcentré du ministère de la Santé), mais hiérarchiquement rattaché au préfet, se voyait confier l'évaluation d'un requérant et était chargé de formuler un avis au préfet $^{2}$.

4 La loi du 11 mai 1998 a ensuite introduit un nouveau mode de régularisation pour l'étranger dont l'« état de santé nécessite une prise en charge médicale dont le défaut pourrait entraîner des conséquences d'une exceptionnelle gravité, sous réserve qu'il ne puisse effectivement bénéficier d'un traitement approprié dans le pays dont il est originaire ». Si cette condition était remplie, la personne bénéficiait de plein droit d'un titre de séjour ${ }^{3}$. La loi Besson du 16 juin 2011 est venu modifier cette condition : elle marque le passage de l'accessibilité des soins à la disponibilité des soins. Dans un arrêt du 7 avril 2010, le Conseil d'État a en ce sens déclaré que si des possibilités de traitements appropriés existent dans le pays d'origine « mais que l'étranger fait valoir qu'il ne peut en bénéficier, soit parce qu'elles ne sont pas accessibles à la généralité de la population, eu égard notamment aux coûts du traitement ou à l'absence de modes de prise en charge adaptés, soit parce qu'en dépit de leur accessibilité, des circonstances exceptionnelles tirées des particularités de sa situation personnelle l'empêcheraient d'y accéder effectivement", l'autorité administrative devra apprécier «si l'intéressé peut ou non bénéficier effectivement d'un traitement approprié dans le pays de renvoi $»^{4}$. Selon la loi Besson, tant que des traitements sont disponibles dans le pays d'origine, l'étranger peut se voir refuser un titre de séjour pour soins. Ainsi la question n'est plus de savoir s'il peut y accéder mais plutôt de savoir si le pays d'origine de l'intéressé dispose des soins nécessaires ${ }^{5}$.

5 Cette loi a enfin été suivie de celle du 7 mars 2016 entrée en vigueur le $1^{\text {er }}$ novembre 2016 qui poursuit trois objectifs principaux dont celui de mieux accueillir et intégrer ceux qui ont le droit de s'établir en France. Elle abroge la loi Besson et le critère de la disponibilité, permettant de revenir au critère de l'accessibilité des soins dans le pays d'origine de l'intéressé. Toutefois, il ne s'agit pas d'un retour au régime prévu par la loi de 1998. En 
effet, il n'est plus question de prendre en compte la situation générale du pays d'origine du requérant mais plutôt la situation propre de ce dernier. Autre changement marquant opéré par la loi de 2016 : l'évaluation médicale se fait désormais à l'OFII. Les médecins sont donc rattachés au ministère de l'Intérieur afin de mieux lutter contre la fraude et contrôler les décisions médicales'.

Le nouvel article 311-6 du CESEDA issu de la loi du 10 septembre 2018 prévoit désormais que «lorsqu'un étranger a présenté une demande d'asile qui relève de la compétence de la France, l'autorité administrative, après l'avoir informé des motifs pour lesquels une autorisation de séjour peut être délivrée et des conséquences de l'absence de demande sur d'autres fondements à ce stade, l'invite à indiquer s'il estime pouvoir prétendre à une admission au séjour à un autre titre et, dans l'affirmative l'invite à déposer une demande dans des conditions fixées par décret... Il est informé que, sous réserve de circonstances nouvelles et sans préjudice des dispositions de l'article L511-4, à l'expiration de ce délai il ne pourra solliciter son admission au séjour ». Cet article vise à empêcher les personnes déboutées de leur demande d'asile de déposer ultérieurement une demande de titre de séjour pour soins 7 . Or il est tout à fait possible, comme le relève le rapport Comede $2017^{8}$, qu'au cours d'un bilan de santé et après le dépôt de la demande d'asile, l'intéressé découvre qu'il souffre d'une maladie concernée par des demandes de titre de séjour pour soins, car les dispositifs de santé sont tout simplement plus accessibles ${ }^{9}$. Les associations dénoncent par conséquent la déconnexion de ce système eu égard à la réalité.

7 Cette évolution du droit au séjour pour soins témoigne de la méfiance du législateur vis-àvis des étrangers malades. C'est ce que confirme aussi la complexification de la procédure de demande de titre de séjour, engendrant en aval une baisse considérable des avis favorables.

\section{II/ - Une complexification de l'accès au titre de séjour}

8 Le déplacement du traitement des personnes étrangères malades des ARS à l'OFII a eu pour corollaire une complexification des procédures d'accès au titre de séjour mention « vie privée et familiale ». Depuis janvier 2017, la procédure commence par le retrait d'un certificat médical sécurisé pour entamer la démarche avec le médecin de la personne étrangère. Avant la réforme, les médecins agréés et praticiens hospitaliers procédaient librement. Désormais, les procédures sont ralenties dès lors que le certificat est mal rempli. L'OFII, dans ce cas, émet immédiatement un avis défavorable au séjour. Dans son rapport de 2017, il souligne que «les délais de traitement des dossiers méritent d'être sensiblement améliorés, même si des délais anormalement longs étaient déjà pointés en $2013 \aleph^{10}$. Or, cet allongement des délais est notamment justifié par la procédure instaurée : un médecin traitant ou un praticien hospitalier établit un certificat médical et l'adresse sous pli confidentiel au service médical de l'OFII, puis, un médecin de l'OFII rédige un rapport médical, à partir duquel un collège à compétence nationale composé de trois médecins émet un avis. C'est cet avis, non couvert par le secret médical, qui est transmis au préfet. La démarche de demande du titre de séjour pour soins fait ainsi intervenir cinq médecins pour chaque dossier.

9 Le rapport de la Cimade ${ }^{11}$ souligne les dangers de ces retards. L'association a constaté sur l'ensemble de l'année 2017 des refus de délivrance de récépissés pour des personnes étrangères malades dans plusieurs préfectures. En effet, selon une circulaire du ministère 
de l'Intérieur du 2 novembre 2016, et une information interministérielle des ministères de l'Intérieur et de la Santé du 29 janvier 2017, les préfectures ne doivent plus délivrer de récépissé aux malades étrangers au moment de l'enregistrement de leur demande en préfecture. La délivrance doit avoir lieu lors de la réception par le collège de médecins de l'OFII du rapport médical rempli par un de ses médecins instructeurs. ${ }^{12}$

Outre le fait que l'attente du rendez-vous à la préfecture dure en général plusieurs mois, certaines délivrent seulement des "attestations de dépôt" qui n'ont aucune valeur légale. Ces personnes étrangères perdent alors pendant quelques mois le droit de travailler, de séjourner, de percevoir certaines prestations et se retrouvent ainsi dans une situation de particulière fragilité. Après plusieurs cas similaires portés devant la justice, le ministère de l'Intérieur a, le 4 mai 2018, publié un décret ${ }^{13}$, demandant de délivrer plus rapidement le récépissé aux personnes renouvelant leur titre de séjour. En l'absence de présentation aux contrôles réalisés à l'OFII sur les personnes malades, les préfectures peuvent refuser de délivrer le récépissé tandis que le droit commun prévoit que la remise d'un récépissé se fait dès le dépôt de la demande.

11 Outre la complexification de la procédure qui peut déjà être perçue comme dissuasive, le taux d'avis favorables au maintien en France a largement diminué. Il passe de $75 \%$ en 2013, après évaluation par les ARS sous la tutelle du ministère de la Santé ${ }^{14}$, à désormais $52 \%$ pour $2017^{15}$. Tandis que le nombre de demandes introduites chaque année d'année ne varie pas, environ 40000 , la France a délivré 4187 titres de séjour à des personnes gravement malades en 2017 contre presque 7000 en $2016^{16}$. Malgré la supposée confiance qu'auraient les préfectures dans les avis médicaux après la réforme, certaines préfectures, comme celle du Doubs, continuent à procéder à des contre-enquêtes pour vérifier leur véracité. Dans d'autres cas, c'est au nom de l'ordre public que, malgré la loi, un préfet décide de passer outre la protection de la santé d'une personne, notamment à la suite du prononcé de peines d'emprisonnement ${ }^{17}$.

12 L'ensemble de ces précisions invite à constater une complexification certaine de la procédure d'obtention d'un titre de séjour pour soin. Certes, selon l'OFII, la France disposerait de l'une des législations les plus favorables d'Europe aux personnes étrangères gravement malades. Toutefois, dans certains pays européens comme la Suisse, même si le taux d'avis favorables est moins important ${ }^{18}$, le titre de séjour est permanent et conduit en conséquence à davantage de sécurité que le titre de séjour français soumis à une obligation de renouvellement annuel.

\section{III/ - Une procédure centrée sur les risques de fraude}

13 Selon le rapport de l'OFII, la chute des délivrances de titres de séjour s'explique par l'effet conjugué de différentes mesures telles que l'amélioration de la qualité des informations médicales, la collégialité des avis ou encore la formation spécifique des médecins de l'OFII. Cependant, au-delà de ces aspects, il importe de noter que le rapport accorde une importance primordiale aux risques de fraude, et ce alors même que les statistiques du rapport montrent que sur environ 40000 demandes traitées, seules 115 représentaient des cas de «fraude avérée ».

Comme cela avait été annoncé par le rapport IGA-IGAS de 2013 et les débats parlementaires de 2015 sur la loi du 7 mars 2016, le rapport érige cette lutte contre la fraude en objectif de la réforme, au même titre que la refonte de l'ensemble du dispositif 
médical d'aide à la décision. Il concède notamment que, « au-delà de la détection de cas avérés ", il s'agit surtout de " prévenir les tentatives de détournement de la procédure » ${ }^{19}$. Cependant cette nouvelle procédure constitue également une difficulté rencontrée par l'OFII. En effet celle-ci étant nouvelle, il a fallu un certain temps d'adaptation aux services, ce qui s'est traduit par un accroissement des délais de traitement des dossiers et un nécessaire investissement en matière de formation du personnel médical.

Cette préoccupation de lutte contre la fraude se retrouve formellement puisque de nombreuses parties du rapport de l'OFII y sont consacrées. Celui-ci consacre par ailleurs un article sur les cas de fraudes qui " sévissaient » sous l'ancienne procédure, celles « des filières recourant au stratagème de fraude $»^{20}$, permettant ainsi de mettre en avant les améliorations apportées. Pour éviter qu'une personne présente les résultats d'examen d'une autre gravement malade, les demandeurs sont soumis à une consultation médicale supplémentaire et exhaustive avec des examens complémentaires tels que des analyses biologiques. Sur l'ensemble des demandes, 50 \%, soit 18146 personnes, ont dû effectuer une consultation médicale supplémentaire.

Un dispositif «d'identitovigilance » est en outre mis en place : la photo du demandeur doit être prise en préfecture et figurer sur le certificat médical vierge à faire remplir par le médecin ; avant l'examen médical de l'OFII, un agent réalise un contrôle d'identité du malade. Ces modifications de procédure entraînent de grandes difficultés pour les personnes malades (allers-retours entre la préfecture et l'OFII de nature à retarder l'accès aux soins ${ }^{21}$. De même les convocations à des examens médicaux supplémentaires peuvent poser problème pour les personnes qui ont une simple domiciliation postale (risque de ne pas être informées à temps et rater leurs convocations). La CIMADE dans son dossier Soigner ou suspecter présente en ce sens plusieurs témoignages des problèmes rencontrés et dénonce une convocation « interrogatoire » plus qu'une convocation médicale ${ }^{22}$.

Si l'OFII a eu l'occasion de rappeler son indépendance et d'affirmer qu'il «ne reçoit aucune instruction ministérielle sur une gestion des flux migratoires $»^{23}$, il est possible de s'interroger sur les similitudes des dispositifs à l'œuvre avec les politiques actuelles de l'OFPRA qui s'est aussi engagé dans une "chasse aux faux réfugiés». Ces courants s'inscrivent dans une volonté politique plus générale de réduction d'accord des titres de séjour.

\section{IV/ - La procédure en centre de rétention}

18 À l'occasion de la réforme de 2016, l'un des enjeux centraux revendiqués par les associations était le renforcement de la protection contre leur expulsion des personnes malades placées en rétention, afin de permettre l'évaluation de leur état de santé. L'obsession de la fraude a néanmoins conduit à opposer un net refus à cette sollicitation, au prétexte que des étrangers pourraient utiliser un tel dispositif dans le but de retarder ou d'empêcher leur expulsion. Comme le souligne le rapport publié par la CIMADE $^{24}$, rien n'a changé dans les centres de rétention depuis que l'OFII est en charge de l'évaluation des personnes étrangères malades. Ces personnes peuvent toujours être expulsées à tout moment pendant l'examen de leur situation médicale. Le rapport de l'OFII expose pourtant qu'il existe des procédures de protection contre l'éloignement, qui se caractérisent par « des délais de traitement plus contraints et un processus de traitement différent selon la nature de l'éloignement $»^{25}$. Lorsque les étrangers, sous mesure d'éloignement placés en centre de rétention administrative s'opposent aux mesures 
d'éloignement en invoquant leur état de santé, un certificat médical est établi par le médecin du lieu de rétention. L'OFII expose que le seul médecin coordonnateur de zone peut émettre un avis sans souligner que, selon la procédure classique, cet avis incombe à un collège de médecins. Cette procédure non-collégiale vise à traiter rapidement les demandes selon l'explication apportée par une information interministérielle de $2017^{26}$.

L'absence de collégialité peut néanmoins être critiquée. Le fait que cette indication soit présentée sans le moindre commentaire dans le rapport peut par ailleurs surprendre étant donné que le rapport de l'OFII justifie la légitimité de la nouvelle procédure amenée par la réforme de 2016 par le constat d'un système antérieur ayant " montré ses limites " d'après un rapport IGA/IGAS de mars $2013^{27}$. Ce rapport de 2013 pointait précisément la " solitude décisionnelle des " médecins des ARS, « l'absence de collégialité dans les avis », et prônait «l'équité de traitement des étrangers demandeurs " ${ }^{28}$. Face à cela, l'OFII explique, d'une part, que la mise en œuvre du dispositif qui lui a été confié est fondée sur les constats de la mission IGA/IGAS, mettant en avant le bénéfice de la collégialité pour la qualité et la solidité des avis, tout en affirmant laconiquement d'autre part, que dans les centres de rétention, l'avis est émis " par le seul médecin coordonnateur de zone ${ }^{29}$. Bien que l'on comprenne aisément l'enjeu de rapidité et d'efficacité du fonctionnement des centres de rétention, le problème repose sur le fait que cette rapidité est priorisée au détriment de la qualité de l'examen de la demande de l'étranger malade.

En 2017, le taux d'avis favorables pour les étrangers retenus était, selon le rapport, de $19,4 \%^{30}$. On conçoit donc aisément que l'une des revendications majeures des organisations à l'occasion de la réforme de 2016 ait été la protection des personnes malades en centre de rétention. Rien n'a changé sur ce point. Et l'augmentation de la durée de rétention prévue par la loi « Asile et immigration » de 2018 ne fera que dégrader la situation des personnes malades dans les centres de rétention. Ce phénomène s'inscrit dans la démarche de lutte contre la fraude... alors même que les rapports recensent seulement 115 cas de fraudes sur les maladies virales ${ }^{31}$, et établissent que la falsification représente $0,5 \%$ des dossiers traités par la délégation territoriale de l'OFII à Bobigny ${ }^{32}$.

Les Lettres "Actualités Droits-Libertés 》 (ADL) du CREDOF (pour s'y abonner) sont accessibles sur le site de la Revue des Droits de l'Homme (RevDH) - Contact

\section{NOTES}

1. \#SoignerOuSuspecter? Le rapport de l'Ofii donne sa réponse, 16 novembre 2018, La CIMADE dans « LE BLOG DE LA CIMADE ».

2. Sur ce point, v. not Klausser, N., «L'étranger gravement malade : un statut fragmenté pour des garanties augmentées ", Revue des droits de l'Homme, n 15, 2019, en ligne. V. encore Klausser, N., «La régularisation pour soins des étrangers : symptômes d'une pathologisation d'un droit de 
l'homme ", Revue des droits de l'Homme, $\mathrm{n}^{\circ} 11$, 2017, accessible en ligne https:// journals.openedition.org/revdh/2890.

3. Avant cette loi (loi n 98-349 du 11 mai 1998 relative à l'entrée et au séjour des étrangers en France et au droit d'asile) les étrangers malades étaient protégés contre l'expulsion mais n'étaient pas automatiquement régularisés.

4. CE, Sect., 7 avril 2010, n 316625 .

5. Loi n 2011-672 du 16 juin 2011 relative à l'immigration, à l'intégration et à la nationalité.

6. Loi $n^{\circ}$ 2016-274 du 7 mars 2016 relative au droit des étrangers en France. Soulignons qu'en pratique, les préfectures refusaient souvent de délivrer des titres aux étrangers, sans même consulter l'avis des médecins.

7. Art. 311-6 du Code de l'entrée et du séjour des étrangers et du droit d'asile.

8. http://odse.eu.org/L-ODSE-DENONCE-DES-ATTEINTES.

9. Loil $n^{\circ}$ 2018-778 du 10 septembre 2018 pour une immigration maîtrisée, un droit d'asile effectif et une intégration réussie.

10. Rapport au parlement : procédure d'admission au séjour pour soins (premier rapport établi en application de l'article L.313-11 $\left(11^{\circ}\right)$ du code de l'entrée et du séjour des étrangers et du droit d'asile), 13 novembre 2018, OFII (Office français de l'immigration et de l'intégration).

11. Soigner ou suspecter?, 21 juin 2017, CIMADE.

12. AIDES Rapport Discriminations 2017, Chapitre 3.

13. Décret $n^{\circ} 2018-335$ du 4 mai 2018 portant modification de dispositions relatives au droit au séjour des étrangers et du décret $n^{\circ} 2015-1423$ du 5 novembre 2015 modifié relatif aux exceptions à l'application du droit des usagers de saisir l'administration par voie électronique.

14. Diéderichs O., Vernhes M., Fournalès R., Chièze F., Rapport sur l'admission au séjour des étrangers malades, Inspection Générale de l'Administration - Inspection Générale des Affaires Sociales, mars 2013.

15. Rapport au parlement : procédure d'admission au séjour pour soins, 13 novembre 2018, OFII, p. 12.

16. Soigner ou suspecter ?, 21 juin 2017, CIMADE.

17. Ibidem.

18. Selon le même rapport de l'OFFI, en 2012, la Suède a attribué 1060 permis de séjour humanitaires sur un total de 12576 demandes d'asile (8,4 \%).

19. Rapport au parlement : procédure d'admission au séjour pour soins (premier rapport établi en application de l'article L.313-11 $\left(11^{\circ}\right)$ du code de l'entrée et du séjour des étrangers et du droit d'asile), 13 novembre 2018, OFII (Office français de l'immigration et de l'intégration).

20. Ibidem.

21. Par exemple la Cimade dans son rapport fournit le témoignage d'une femme réalisant les formalités pour son fils et perdant tous ses droits sociaux du fait d'une simple erreur de nom ayant entraîné la non-conformité du certificat médical.

22. \#SoignerOuSuspecter? Le rapport de l'Ofii donne sa réponse, 16 novembre 2018, La CIMADE dans « LE BLOG DE LA CIMADE ».

23. Post Facebook du 16 novembre.

24. Soigner ou suspecter?, 21 juin 2017, CIMADE (Comité Inter-Mouvements Auprès Des Évacués) p. 25.

25. Ibidem p. 25

26. Information du 29 janvier 2017 relative à l'application de la loi $n^{\circ} 2016-274$ relative au droit des étrangers en France (dispositions relatives à la procédure de délivrance des documents de séjour et à la protection contre l'éloignement pour raison de santé, applicables à compter du 1er janvier 2017. 
27. Diéderichs O., Vernhes M., Fournalès R., Chièze F., Rapport sur l'admission au séjour des étrangers malades, Inspection Générale de l'Administration - Inspection Générale des Affaires Sociales, mars 2013.

28. Ibid. p. 17.

29. Ibid. p. 21.

30. Ibid. p. 21.

31. Ibid. p. 39.

32. Ibid. p. 40.

\section{ABSTRACTS}

La situation des étrangers demandant des titres de séjour pour soins a changé le 7 mars 2016 avec la loi $n^{\circ}$ 2016-274 relative au droit des étrangers en France. Alors que la loi avait pourtant pour objectif de simplifier les procédures pour mieux subvenir aux besoins des étrangers malades en centre de rétention, force est de constater, à l'issue des bilans de cette loi réalisés par la CIMADE et l'Office français de l'immigration et de l'intégration: une baisse considérable des avis favorables, une complexification de la procédure, une logique de dissuasion des étrangers gravement malades à formuler une demande ainsi que la primauté octroyée à la lutte contre la fraude au détriment de la prise en charge. In fine, est consacrée une tendance générale dangereuse, tant pour la santé publique que pour les individus malades.

\section{AUTHORS}

\section{AUDE FRAYSSE}

Étudiante du M2 Droits de l'Homme à l'Université Paris Nanterre

\section{ESTHER LANEELLE}

Étudiante du M2 Droits de l'Homme à l'Université Paris Nanterre

\section{YASMINE HAMMADI}

Étudiante du M2 Droits de l'Homme à l'Université Paris Nanterre

\section{CLOTHILDE LAGORSSE}

Étudiante du M2 Droits de l'Homme à l'Université Paris Nanterre

\section{RITA MBEN}

Étudiante du M2 Droits de l'Homme à l'Université Paris Nanterre 